\title{
Evaluation of brain edema formation defined by MRI after LINAC-based stereotactic radiosurgery
}

\author{
Maciej Harat ${ }^{1}$, Andrzej Lebioda²,4, Judyta Lasota ${ }^{3}$, Roman Makarewicz 2,4 \\ ${ }^{1}$ Department of Radiotherapy, The Franciszek Lukaszczyk Oncology Center, Bydgoszcz, Poland \\ 2 Department of Oncology and Brachytherapy, Nicolaus Copernicus University, Ludwik Rydygier Collegium Medicum, \\ Bydgoszcz, Poland \\ ${ }^{3}$ Department of Medical Physics, The Franciszek Lukaszczyk Oncology Centre, Bydgoszcz, Poland \\ ${ }^{4}$ Department of Oncology and Brachytherapy, The Franciszek Lukaszczyk Oncology Center, Bydgoszcz, Poland
}

Radiol Oncol 2017; 51(2): 137-141.

Received 28 August 2016

Accepted 2 March 2017

Correspondence to: Maciej Harat, 85-796 Bydgoszcz, Romanowskiej 2 St., Poland. Phone: +48 52374 3479; E-mail: haratm@co.bydgoszcz.pl Disclosure: No potential conflicts of interest were disclosed.

Background. Peri-lesional edema is a serious and well-known complication of stereotactic radiosurgery (SRS). Here we evaluated edema risk after SRS and assessed its formation and resolution dynamics.

Patients and methods. 107 patients underwent SRS for heterogeneous diagnoses: 34 (29\%) with arteriovenous malformations, 38 (35\%) with meningiomas, 16 (15\%) with metastatic tumors, 16 (15\%) with acoustic neuromas, 3 with (3\%) cavernomas, and $2(2 \%)$ each with anaplastic astrocytomas and anaplastic oligoastrocytomas. Edema area was delineated in MRI T2-FLAIR sequences $0,6,12,18,24,30$, and 38 months after treatment. Lesion location was defined as either above $(n=80)$ or below $(n=32)$ the "Frankfurt modified line" (FML).

Results. $17 \%$ of patients developed or had worsening post-treatment edema. Edema volume was maximal at 6 months (mean 7.2, SD 1.2) post radiosurgery. Post-SRS edema was 5.1 (1.06 - 24.53) times more likely in patients with lesions above the FML. There was no association between edema development and age, PTV size, number of beams, and diagnosis ( $\mathrm{p}=0.07$ ).

Conclusions. Radiosurgery-associated edema develops within 6 months of treatment and decreases over time. Edema occurrence is strongly related to lesion location, and its presence is much more likely when the treated lesions are situated above the Frankfurt line.

Key words: brain edema; LINAC; radiosurgery; stereotactic

\section{Introduction}

Intracranial stereotactic radiosurgery (SRS) is a radiotherapy technique used to treat patients unsuitable for surgery or after partial resection of meningiomas, neuromas, brain metastases, and vascular malformations. Typical eligibility criteria are patients with tumors not exceeding $3.5 \mathrm{~cm}$ and those who can cooperate during the procedure. ${ }^{1,2}$

Brain edema is a common radiosurgery-related side effect ${ }^{3-7}$ that often requires symptomatic treat- ment since untreated it can impair quality of life or even cause death. For many years, the most effective edema treatments have been corticosteroids (dexamethasone) and mannitol.

The exact pathophysiology of edema formation after radiotherapy remains unclear but may be related to cerebrovascular impairment, disruption of the blood-brain barrier, or radiation-induced damage to microglia and astrocytes. ${ }^{8}$ Aquaporin 4 and other mediators have been implicated in edema formation including inflammatory cytokines, an- 
giogenesis factors (VEGF), hypoxia-related factors (HIF-1), cyclooxygenases, and markers of glial activation. $5,9-11$

Current evidence suggests that radiosurgeryrelated brain edema is associated with tumor size, location of the lesion, the prescribed radiotherapy dose, and the presence of edema before treatment. ${ }^{12,13}$ Some data show that brain edema, even in homogenous clinical groups, is unrelated to the maximum dose or the surrounding conformity index..$^{14}$ It should be noted that most existing research concerns edema related to meningioma radiosurgery. $5,12,13$

We have observed edema after radiosurgery in patients with various clinical and histopathologic diagnoses in our practice, the significance of which remains uncertain. We therefore sought to identify factors that predispose to edema formation after SRS and assess edema formation and resolution dynamics.

TABLE 1. Baseline patient characteristics

\begin{tabular}{|c|c|c|}
\hline \multicolumn{2}{|l|}{ Characteristic } & \multirow{2}{*}{$\begin{array}{c}\begin{array}{c}\text { Number o } \\
\text { patients }\end{array} \\
111\end{array}$} \\
\hline Total number of lesions & & \\
\hline \multirow[t]{2}{*}{ Sex } & Male & 40 \\
\hline & Female & 63 \\
\hline Age (mean) & & 51 \\
\hline \multirow[t]{7}{*}{ Histopathological diagnosis } & Arteriovenous malformation & 34 \\
\hline & Meningioma & 38 \\
\hline & Metastasis & 16 \\
\hline & Neuroma & 16 \\
\hline & Cavernous angioma & 3 \\
\hline & Anaplastic astrocytoma & 2 \\
\hline & Anaplastic Oligoastrocytoma & 2 \\
\hline \multirow[t]{11}{*}{ Lesion location } & Ponto-cerebellar angle & 24 \\
\hline & Cerebellum & 7 \\
\hline & Parietal lobe & 16 \\
\hline & Frontal lobe & 17 \\
\hline & Subcortical nucleus/hippocampus & 12 \\
\hline & Convexity & 2 \\
\hline & Petroclival & 1 \\
\hline & Temporal lobe & 8 \\
\hline & Falx & 13 \\
\hline & Tentorium & 3 \\
\hline & Occipital lobe & 4 \\
\hline $\begin{array}{l}\text { More than one SRS } \\
\text { treatment }\end{array}$ & & 8 \\
\hline Mean $V_{12}$ & & $6.3 \mathrm{~cm}^{3}$ \\
\hline
\end{tabular}

\section{Patients and methods}

One hundred and three patients undergoing 111 SRS procedures were treated in the Oncology Centre in Bydgoszcz between January 2008 and October 2012. The study population had a heterogeneous set of histopathological diagnoses: 34 $(29 \%)$ arteriovenous malformations (AVMs), 38 $(35 \%)$ meningiomas, $16(15 \%)$ metastatic tumors, $16(15 \%)$ neuromas, $3(3 \%)$ cavernous angiomas, 2 (2\%) anaplastic astrocytomas, and $2(2 \%)$ anaplastic oligoastrocytomas. The baseline patient characteristics are shown in Table 1.

Patients were treated with a $6 \mathrm{MV}$ photon beam provided by a linear accelerator (Varian, USA) and a micromultileaf collimator with $3 \mathrm{~mm}$ width leaves at the isocenter (Brainlab, Germany). The median prescribed doses at the lesion margin (isodose line) was $16 \mathrm{~Gy}$ for all lesions apart from for neuromas (12 Gy). Patient immobilization and target volume definition were achieved using a stereotactic frame or thermoplastic mask. After mask/frame fixation, three-dimensional computed tomography (3D-CT) was performed and then fused with non-stereotactic gadolinium-enhanced T1-weighted magnetic resonance imaging (MRI) sequences.

All patients with radiological signs of brain edema during follow-up examinations were included. Edema volume was delineated on T2-weighted axial MRI/FLAIR scans with a $1 \mathrm{~mm}$ slice thickness using the Oncentra Brachy (Nucletron) treatment planning workstation. Edema analysis was performed on MRI scans prior to treatment and 3, 6, $9,12,18,24,30$, and 36 months after treatment. An example is shown in Figure 1.

To analyze lesion location, lesions were separated into occurrence above $(n=80)$ and below $(n=32)$ a plane separating the top and bottom of the skull extending from frontal to parietal (protuberatnia frontalis to protuberantia occipitalis) and situated $3 \mathrm{~cm}$ above the Frankfurt line ${ }^{14}$ (referred to here as the Frankfurt modified line (FML)) (Figure 2). All infratentorial tumors (number), lesions localized in the central brain structures (e.g., hippocampus, caudate nucleus), lesions localized to the temporal and inferior parts of the frontal lobes, and meningiomas of the tentorium and sella turcica were regarded as "below" FML lesions and all others as "above" FML lesions. The study was performed in accordance with the principles of the Helsinki Declaration and was approved by the Ethics Committee of Collegium Medicum of Nicolaus Copernicus University. 

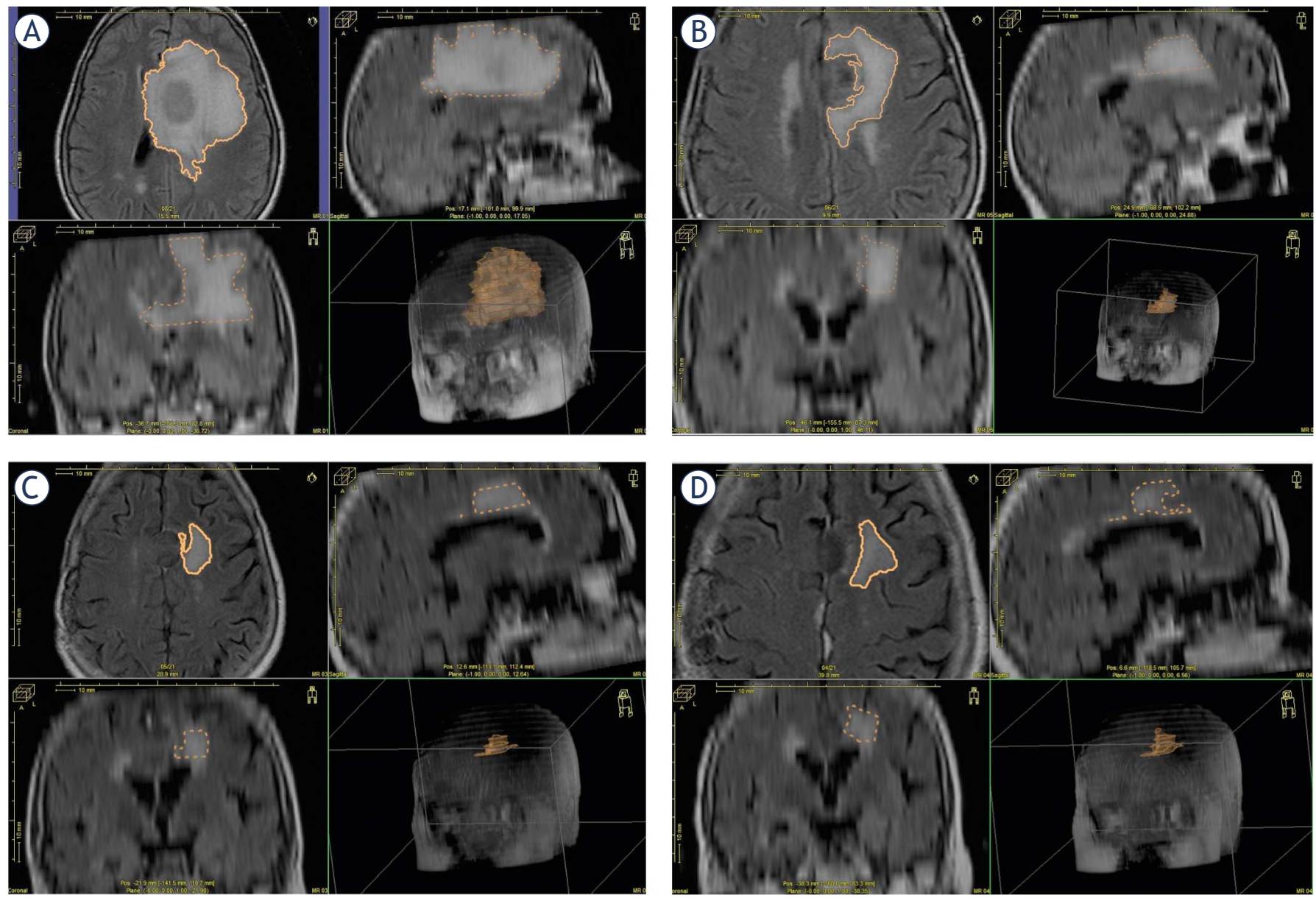

FIGURE 1. MRI scans performed after treatment showing delineation of area with decreasing edema. (A) 6 months; (B) 12 months; (C) 18 months; (D) 24 months.

All statistical analyses were performed using Statistica v.10 (Dell Inc., Austin, TX).

\section{Results}

Five patients did not attend for follow-up studies and were excluded from further analysis. SRSassociated edema occurred in 17 patients, of whom one patient was diagnosed with a meningioma located in the pontocerebellar angle with edema prior to SRS and that increased in volume in the six months after treatment. The initial MRI did not reveal edema in the other patients. SRS-related edema occurred in 2/16 (12.5\%) metastatic tumors, 5/34 (14.7\%) AVMs and 10/38 (26.3\%) meningiomas.

The dynamics of edema formation over time (log-normal distribution) are shown in Figure 3. There were no associations between edema development and age, size of irradiated area, volume of

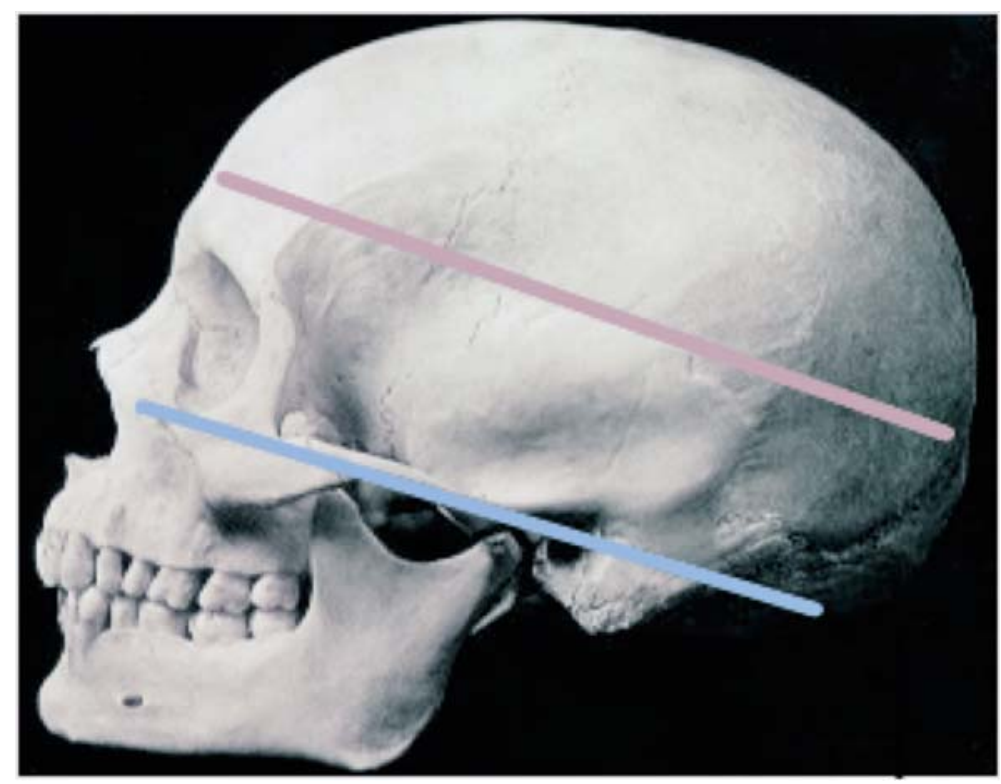

FIGURE 2. The Frankfurt Line (blue line) and the Frankfurt modified line (FML; red line). 


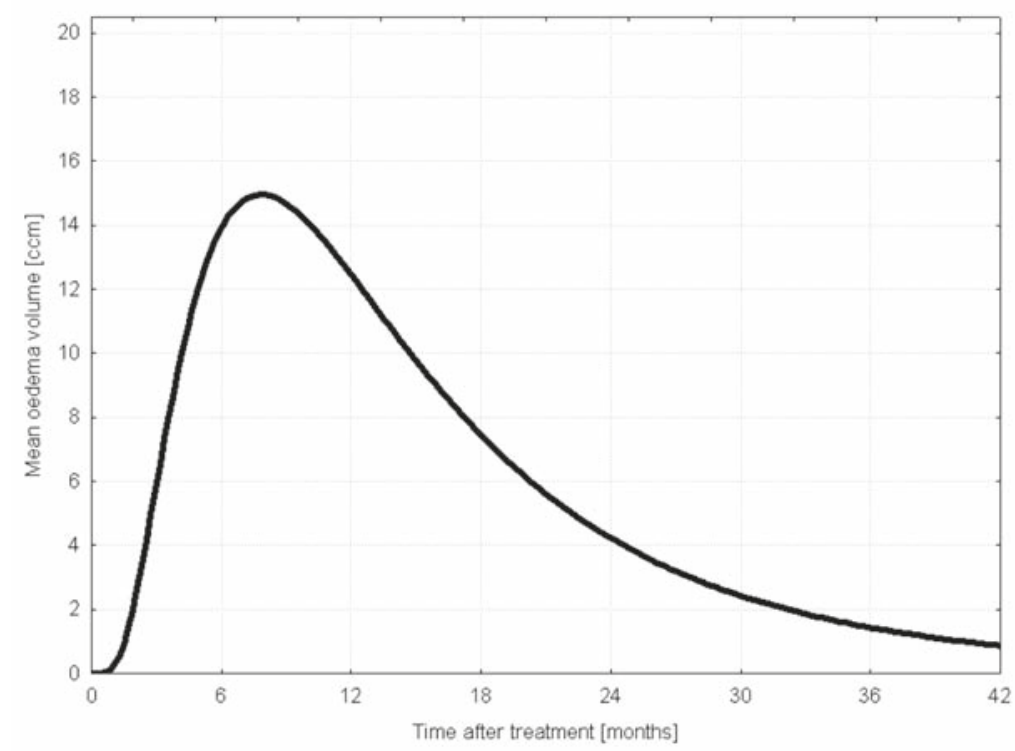

FIGURE 3. The largest volume of edema was observed at 6 months (mean 7.2 months, S.D. 1.2 months) after radiosurgery and decreased thereafter.

normal brain covered by $12 \mathrm{~Gy}$ isodose $\left(\mathrm{V}_{12}\right)$, number of beams, type of tumor $(p=0.07)$, or the type of immobilization applied.

With respect to lesion location, edema occurred in 2/32 lesions below the FML and in 15/80 lesions above the FML, representing a 5.1-times (1.06 24.53 ) increased incidence of edema after radiosurgery for lesions above the FML.

There was no association between edema development after secondary radiosurgery in the same patients if no edema formed after the initial treatment.

\section{Discussion}

Radiosurgery-related peri-lesional edema is a wellknown but rarely described complication of SRS. Here we present the relationship between edema occurrence and lesion location in a population of patients with heterogeneous diagnoses.

The incidence of edema in this cohort $(17 \%)$ is consistent with the published literature, recognizing that most data relates to edema occurring after radiosurgery for meningiomas. Chang et al. observed edema in $26.3 \%$ of patients with meningiomas after radiosurgery, about $40 \%$ of whom presented with symptoms of increased intracranial pressure. ${ }^{15}$ Kobayashi et al. reported edema in $13.8 \%$ of patients with benign meningiomas after radiosurgery. ${ }^{16}$ With respect to radiosurgery-relat- ed edema in patients with other histopathological diagnoses, Hallemeier et al. reported edema after radiosurgery in $44 \%$ of patients with astrocytoma, which was effectively treated with oral or intravenous steroid therapy ${ }^{17}$, while Williams et al. described a case of steroid-resistant edema treated with bevacizumab that developed 9 months after radiosurgery for a vascular malformation. ${ }^{18}$

Cai et al. found that the risk of edema increased with greater tumor-brain contact interface area, supporting the observed differing incidence of edema according to location: the decreased incidence of edema below the FML may be associated with the amount of brain surrounding the lesion, since the lesion-brain contact interface above the FML is greater than below the FML. ${ }^{19}$

We found no association between the presence of edema and lesion size, dose escalation, or histopathological diagnosis. Although this finding may be due to the small number of patients in each group, these parameters need to be taken into account when assessing edema risk after radiosurgery. $^{20}$

The lack of an association between the histopathological diagnosis and lesion size suggests a common pathway for post-SRS edema formation, most likely related to impaired blood flow surrounding the intracranial lesion and explaining why the incidence of edema is lower in locations with better blood supply. For instance, with respect to tumors occurring in the parasagittal region, a higher edema risk may be present due to an unfavorable location (above the FML) and a histopathological diagnosis of meningioma. In this area, numerous veins carry blood to the superior sagittal sinus and the lower and deep vein (Galena vein), and numerous vessels at the tumor border drain blood from the tumor to normal brain vessels. Radiosurgery may impair flow in these vessels, similar to the mechanism seen when vessels in an AVM are obliterated. This leads to regional impairment of venous drainage from the brain and, as a consequence, edema.

We observed an increase in edema volume over the first $6-9$ months and a decrease thereafter. In one retrospective multi-center study, the average duration of edema after radiosurgery was estimated to be 15 months (range $5-18$ months) ${ }^{21}$, with maximum accumulation after $6-8$ months, consistent with the results presented here. Not all brain edema is symptomatic or requires treatment, but if edema is observed radiologically at followup 3 months after treatment even in the absence of symptoms, a short regimen of steroids may be ap- 
propriate to avoid the natural dynamics of edema formation occurring.

Patients treated with multiple rounds of radiosurgery to more than one lesion are a valuable study group for assessing the prevalence of complications. Here, this patient subgroup did not develop edema at follow up after both the first and subsequent treatments. This may suggest that resistance to edema formation depends on host or individual factors, but this hypothesis would require further clinical validation.

The study has several limitations. The FML plane was arbitrarily selected by the investigators from personal observations and does not represent an anatomical border that may explain differences in edema formation. Due to the retrospective nature of the analysis, we were unable to examine steroid treatment and its influence on edema formation and resolution. Nevertheless, to our knowledge, this is one of the largest studies of SRS-related edema formation.

\section{Conclusions}

Peri-lesional cerebral edema is a temporary intracranial complication after radiosurgery. The most severe MRI changes can be observed in the first six to nine months after treatment. The occurrence of cerebral edema is dependent on the intracranial location. Localization of lesion above the FML is a strong predictor of the emergence of this treatment complication.

\section{Acknowledgements}

We gratefully acknowledge Nextgenediting (www. nextgenediting.com) for editorial assistance.

\section{References}

1. Chin L, Regine W. Principles and practice of stereotactic radiosurgery. New York: Springer; 2008.

2. Bakay RA. Stereotactic radiosurgery in the treatment of brain tumors. Clin Neurosurg 1992; 39: 292-313.

3. Nakamura S, Hiyama H, Arai K, Nakaya K, Sato H, Hayashi M, et al. Gamma knife radiosurgery for meningiomas: four cases of radiation-induced edema. Stereotact Funct Neurosurg 1996; 66: 142-5. doi: 10.1159/000099804

4. Ganz JC, Schrottner O, Pendl G. Radiation-induced edema after gamma knife treatment for meningiomas. Stereotact Funct Neurosurg 1996; 66: 129-33. doi: $10.1159 / 000099778$

5. Kan P, Liu JK, Wendland MM, Shrieve D, Jensen RL. Peritumoral edema after stereotactic radiosurgery for intracranial meningiomas and molecular factors that predict its development. I Neurooncol 2007; 83: 33-8. doi: $10.1007 /$ s11060-006-9294-y
6. Morimoto $\mathrm{M}$, Yoshioka $\mathrm{Y}$, Shiomi H, Isohashi F, Konishi K, Kotsuma T, et al. Significance of tumor volume related to peritumoral edema in intracranial meningioma treated with extreme hypofractionated stereotactic radiation therapy in three to five fractions. Jpn J Clin Oncol 2011; 41: 609-16. doi: 10.1093/jjco/hyr022

7. Unger KR, Lominska CE, Chanyasulkit J, Randolph-Jackson P, White RL, Aulisi E, et al. Risk factors for posttreatment edema in patients treated with stereotactic radiosurgery for meningiomas. Neurosurgery 2012; 70: 639-45. doi: 10.1227/NEU.0b013e3182351ae7

8. Papadopoulos MC, Saadoun S, Binder DK, Manley GT, Krishna S, Verkman AS. Molecular mechanisms of brain tumor edema. Neuroscience 2004; 129: 1011-20. doi: 10.1016/j.neuroscience.2004.05.044

9. Saadoun S, Papadopoulos MC. Aquaporin-4 in brain and spinal cord oedema. Neuroscience 2010; 168: 1036-46.

10. Moore AH, Olschowka JA, Williams JP, Paige SL, O'Banion MK. Radiationinduced edema is dependent on cyclooxygenase 2 activity in mouse brain. Radiat Res 2004; 161: 153-60.

11. Khan RB, Krasin MJ, Kasow K, Leung W. Cyclooxygenase-2 inhibition to treat radiation-induced brain necrosis and edema. J Pediatr Hematol Oncol 2004; 26: 253-5.

12. Chang JH, Chang JW, Choi JY, Park YG, Chung SS. Complications after gamma knife radiosurgery for benign meningiomas. I Neurol Neurosurg Psychiatry 2003; 74: 226-30

13. Kalapurakal JA, Silverman CL, Akhtar N, Laske DW, Braitman LE, Boyko OB, et al. Intracranial meningiomas: factors that influence the development of cerebral edema after stereotactic radiosurgery and radiation therapy. Radiology 1997; 204: 461-5. doi: 10.1148/radiology.204.2.9240536

14. Florentyna Łabiszewska-Jaruzelska: Ortodoncja: zasady i praktyka. Warszawa: PZWL; 1995, p. 57.

15. Chang JH, Chang JW, Choi JY, Park YG, Chung SS. Complications after gamma knife radiosurgery for benign meningiomas. I Neurol Neurosurg Psychiatry 2003; 74: 226-30.

16. Kobayashi T, Kida Y, Mori Y. Long-term results of stereotactic gamma radiosurgery of meningiomas. Surg Neurol 2001; 55: 325-31.

17. Hallemeier CL, Pollock BE, Schomberg PJ, Link MJ, Brown PD, Stafford SL. Stereotactic radiosurgery for recurrent or unresectable pilocytic astrocytoma. Int J Radiat Oncol Biol Phys 2012; 83: 107-12. doi: 10.1016/j. ijrobp.2011.05.038

18. Williams BJ, Park DM, Sheehan JP. Bevacizumab used for the treatment of severe, refractory perilesional edema due to an arteriovenous malformation treated with stereotactic radiosurgery. J Neurosurg 2012; 116: 972-7. doi: 10.3171/2012.1.JNS111627

19. Cai R, Barnett GH, Novak E, Chao ST, Suh JH. Principal risk of peritumoral edema after stereotactic radiosurgery for intracranial meningioma is tumor-brain contact interface area. Neurosurgery 2010; 66: 513-22. doi: 10.1227/01.NEU.0000365366.53337.88

20. Unger KR, Lominska CE, Chanyasulkit JBA, Randolph-Jackson P, White RL, Aulisi $E$, et al. Risk factors for posttreatment edema in patients treated with stereotactic radiosurgery for meningiomas. Neurosurgery 2012; 70: 639-45. doi: 10.1227/NEU.0b013e3182351ae7

21. Kondziolka D, Flickinger JC, Perez B. Judicious resection and/or radiosurgery for parasagittal meningiomas: outcomes from a multicenter review-Gamma Knife Meningioma Study Group. Neurosurgery 1998; 43: 405-14. 